\title{
Exploration and Reform on TCP/IP Protocol Analysis Experiment Under Simulation System
}

\author{
Xu Xiaolin ${ }^{1, \text { a }}$, Liu Yan ${ }^{2, b}$, Wang Jian ${ }^{3, c}$, Si Taozhi, ${ }^{4, d}$
}

1, 2, 3, 4 College of Computer and Information,Shanghai Second Polytechnic University,

\author{
Shanghai,China \\ axlxu2001@163.com, bliuyan@sspu.edu.cn, cwangjian@sspu.edu.cn, dtzsi@sspu.edu.cn
}

Keywords: TCP/IP Protocol; Simulation; Experiment

\begin{abstract}
TCP/IP protocol architecture is complex and difficult to understand. In addition to classroom teaching, practical teaching is also very important.The paper introduces protocol simulation system into practical teaching,illustrates analysis examples of ICMP,IP and ARP packets run under the system and stresses the important roles the system plays on theoretical teaching.
\end{abstract}

\section{Importance of TCP/IP Protocol Experiment Course}

TCP/IP protocol is the suite of communications protocols used to connect hosts on the Internet and provides the most basic mechanism for computer network to achieve its functions. A large number of abstract and complex protocols run at all levels in the computer network architecture,so it is very difficult for students to understand and master the contents only through theoretical teaching.How to improve experimental teaching of TCP/IP protocol by building a simulation platform for network protocol experimental teaching,which enables students to better understand, validate and consolidate classroom teaching contents and enhances their perceptual knowledge on network protocol and thereby cultivates their ability to integrate theory with practice is a problem to be solved ${ }^{[1]}$.

To experiments of the TCP/IP course is Computer Protocol Simulation System from JLCSS applied.Through the system,students can visually observe not only transmission of data streams across the network and protocol conversations,but roles protocols from different levels play in one communication,which enhances students ' understanding of protocols and improves their ability to analyze and solve problems.

\section{Simulation Platform Introduction}

Components. The simulation platform is composed of two functional softwares ${ }^{[2]}$ :Simulation Editor and Protocol Analyzer.

- Simulation Editor

Simulation Editor is used to visually edit some protocol packets of TCP/IP,and after that send them to network.

- Protocol Analyzer

Protocol Analyzer is responsible for capturing frames in the network and analyzing protocols contained in the different layers.It includes two functional modules: Conversation Analysis and Protocol Analysis.

- Conversation Analysis can add captured data frames of commonly used protocols to the list of sessions and shows session sequences and data transmission directions, which enables students to observe and analyze a full session visually and understand in-depth protocol principles and working process.

- Protocol Analysis incoudes the display of outline codes, detailed decoding and initial data,which provides detailed explanation and description of protocols and dynamic tracking display depending on choices so as to analyze data easily.

Experiment Form. Experiment form is to divide hosts into groups.Each group consists of six hosts 
marked A,B,C,D,E,F respectively.There are three topology available for experiment environment.In each topology,six hosts can edit and capture packets acting as different roles to show working process of TCP/IP protocols. $^{[3]}$

\section{Protocol Analysis Teaching Examples Applying Simulation System}

ARP is an auxiliary protocol of IP.When a packet reaches a node,IP will select the path for the being transmitted packet based on its destination IP address to get IP address of the next node.At this point,ARP is responsible for resolving the IP address of the next node into MAC address provided for the frame formed on the layer of data link. It can be seen from this process that ARP acts as a connecting bridge between network layer and data link layer.Studying ARP,students are able to understand the roles played by MAC address and data link layer in the network communication.

This section should be taught by associating it with routing table that describes how IP packets are passed from the perspective of network layer,whereas ARP reflects at a certain extent how data frames are passed at the data link layer.The combination helps students have a relatively complete understanding of the roles IP address and MAC address play during the network transferring and thereby understand the meanings of network layer and data link layer ${ }^{[4]}$.In addition, specific experiment can be used to verify this combination of two points.

With regards to this, a verification experiment is designed with the second topology simulation platform gives, which is shown in Fig 1.

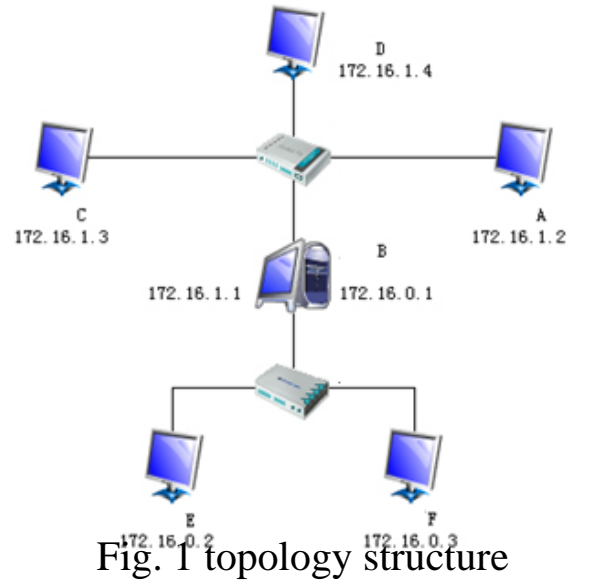

In the topology,Host A,C and D are in the same subnet (172.16.1.0/24) ,and Host $\mathrm{E}$ and $\mathrm{F}$ are in the same subnet (172.16.0.0/24).Host B with two network interface cards functions as a router connected to two subnets after routing configuration. ${ }^{[5]}$

Now Host A (172.16.1.2) Ping Host E

(172.16.0.2) .Since Ping is the application of ICMP,the Ping packet is first encapsulated as ICMP packet and in turn encapsulated as IP packet (shown in Fig.2), and then makes routing according to the routing table of Host

A(as shown in Fig.3).Due to A and $\mathrm{E}$ in the different subnets, $\mathrm{A}$ is not able to directly transfer the IP packet to E.After referring to the routing table, A learns that the packet should be transmitted to the router's interfaces b1 (172.16.1.1). As for specific transmission,the IP packet requires encapsulating in the frame with the corresponding MAC address of 172.16.1.1 as its destination MAC address.Next, ARP is needed to play the role as a bridge.Given that there is no mapping of $\mathrm{MAC}$ address to 172.16.1.1,ARP needs to send a request packet(shown in Fig.4) in which MAC address (008899-000495), IP address (172.16.1.2) of Sender A,and Destination MAC address（000000-000000）,IP address (172.16.1.1) are specified respectively. 


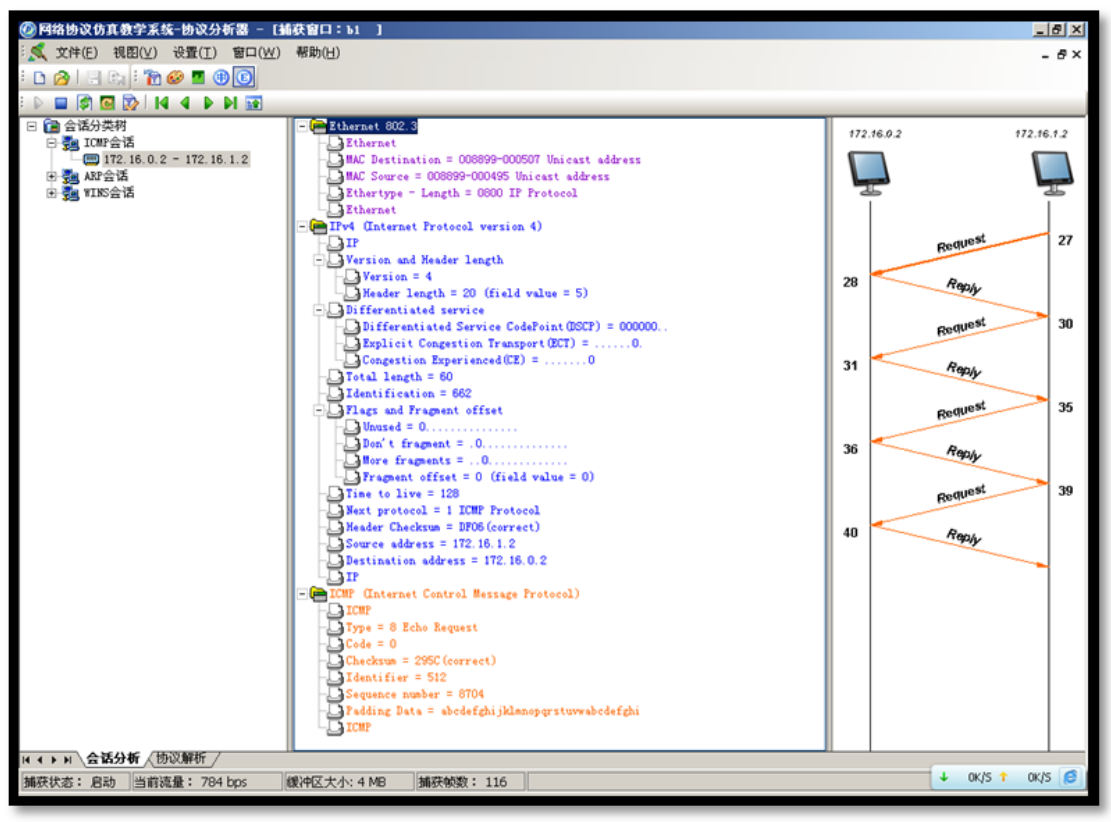

Fig. 2 Encapsulation of ICMP packet

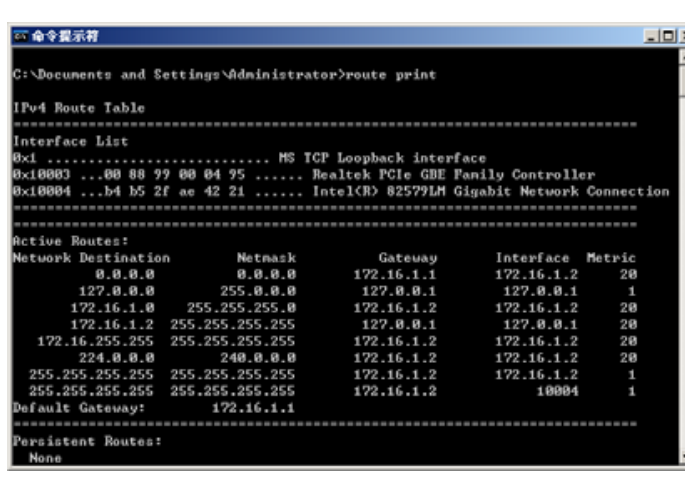

Fig.3 Routing Table of Host A

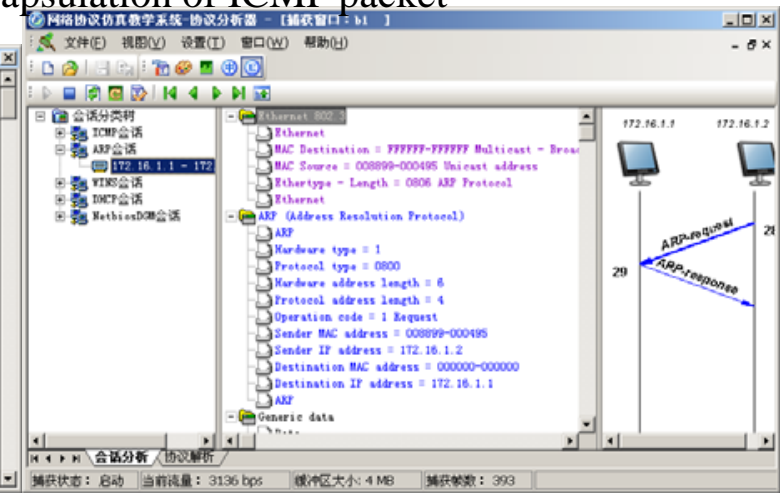

Fig.4 ARP Request Packet Captured by Interface b1 of Host B

The ARP packet is encapsulated in a MAC frame with FFFFFF-FFFFFF destination address meaning that it is broadcasted so as to be received by Host C、D、b1.And only b1 will reply the ARP request to Host A.After receiving the reply,Host A saves the mapping of 172.16.1.1 to 00-88-99-00-05-07 in its cache(shown in Fig.5) and the ARP process is finished.

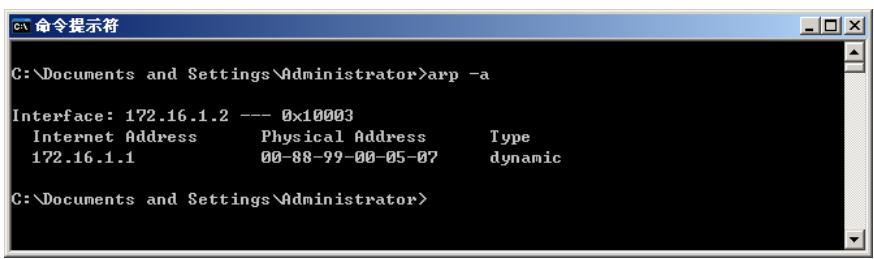

Fig. 5 ARP cache of Host A

After the encapsulated initial ICMP packet reaches Host B,B refers to its routing table to find out destination IP address(172.16.0.2) in IP packet is in the same subnet as its.Therefore the IP packet can be transferred to destination Host E directly. Similarly,the MAC address is required,so ARP works again by sending ARP request packet(shown in Fig.6)through b2 in which MAC address (B4B52F-AB1A23) ,IP address (172.16.0.1) of Sender b2,and destination MAC address ( 000000-000000) ,IP address (172.16.0.2) are specified respectively.Both $\mathrm{E}$ and $\mathrm{F}$ are able to receive the broadcasted packet.E sends the ARP reply packet to b2,and then b2 saves the mapping of 172.16.0.2 to 00-88-99-00-05-6f in its 
cache(shown in Fig.7) and the ARP process is finished.

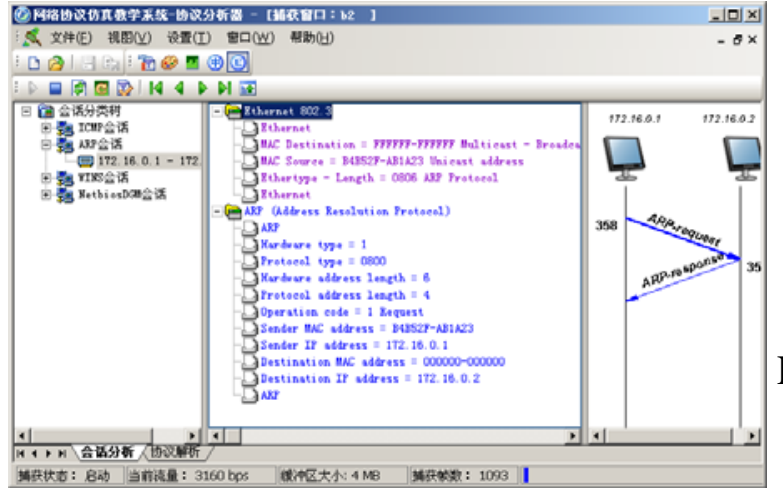

Fig.6 ARP Request Packet Captured by Interface b2 of Host B

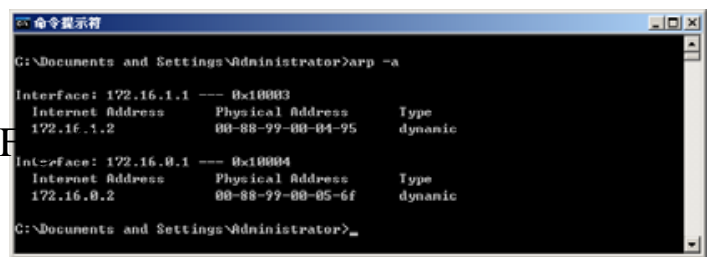

Fig.7 ARP cache of Host B

Now Let's go back to the IP capsulation of original ICMP packet,source IP and destination IP of the IP packet remain unchanged during the transmission, whereas source MAC address and destination MAC address has been changing from node to node, which indicates that IP packets select path based on destination IP to determine the IP address of next hop,and next are encapsulated into frames and transferred to next node.In specific transmission,it is MAC address not IP address that is used to locate and track the flowing of packets.

\section{Summary}

Practice has proved that applying the simulation platform enables students to observe encapsulation and processing of packets and have an active leap from rational to perceptual knowledge in the understanding of abstract concepts and theories,and thus helps them understand better the core ideas of Internet model manifests and prompts them to think in-depth design concepts of TCP/IP protocols and achieves the goal of "Know the what,know the why".

\section{References}

[1] Zhao Xiulan,in: Exploration on Experiment Course of TCP/IP Protocol and Analysis,edited by the Science Education Article Collects,Total 261 (2013).

[2] Ma Li,Yu Helong,Deng Leilei,Wang Jinghui,in:Exploration on Computer Network Experiment Course Based on Network Protocol Simulation Platform,edited by China Education Info,vol.12,(2010).

[3] Xu Xiaolin,Liuyan,Wangjian,in:Thinking of Reform on the Teaching of TCP/IP Protocol, edited by 2nd International Conference on Social Science and Education(2013).

[4] Duo Yunfeng,Wang Yi,Rong Hui,in:Towards an Effective Teaching of TCP/IP Protocol, edited by Journal of Kunming Metallurgy College,Vol.22,No.1(2006).

[5] Guo Dongwei,Zhoubin,Huo Yanmei.in:Emulational Education System for Network Protocols,edited by China Machine Press(2008). 